\title{
Film ou vidéo
}

Réflexions sur la pérennité d'une controverse technique

Film or video, reflexions on the lonevity of a technological controversy

Jérôme Bourdon

\section{(2) OpenEdition}

Journals

Édition électronique

URL : https://journals.openedition.org/tc/5027

DOI : $10.4000 /$ tc. 5027

ISSN : 1952-420X

Éditeur

Éditions de l'EHESS

Édition imprimée

Date de publication : 30 juin 2010

Pagination : 651-669

ISSN : 0248-6016

Référence électronique

Jérôme Bourdon, «Film ou vidéo », Techniques \& Culture [En ligne], 54-55 | 2010, mis en ligne le 30 juin 2013, consulté le 29 septembre 2022. URL : http://journals.openedition.org/tc/5027 ; DOI : https:// doi.org/10.4000/tc.5027 


\title{
Réflexions sur la pérennité d'une controverse technique
}

\author{
in Techniques et culture 16, 1990 : 161-182
}

\section{Mais qúest-ce donc qui les sépare?}

\begin{abstract}
À la fin des années cinquante, s'engage à la télévision française un débat qui dure encore. Faut-il produire les émissions dramatiques en vidéo ou en film? Émissions dramatiques: on dira plus tard les « dramatiques »; on dirait aujourd'hui les « téléfilms » et les « feuilletons »- le feuilleton ne venant s'ajouter à la palette des genres qu'à la fin des années cinquante. Voilà notre sujet, et, contrairement à la règle académique qui prescrit de dire avec précision les limites avant d'explorer le territoire, nous devons nous en tenir à cette question vague. L'intérêt tient précisément à la difficulté de la formuler en termes plus précis. Le faire serait entrer plus avant dans la controverse, c'est-à-dire prendre position. Un exemple: nous avons été tenté de dire "s'engage entre ingénieurs et réalisateurs ", supposant ainsi définis deux groupes professionnels qui vont jouer un rôle essentiel dans la controverse, mais qui vont aussi être partiellement définis par elle. Loin d'être un a priori, de nous offrir des cultures de métier qui expliqueraient à leur tour un point de vue sur les objets techniques, la définition des groupes « professionnels » fait partie du problème que nous nous posons. Un des enjeux de la controverse, c'est la définition et la mobilisation de groupes supposés prendre le parti du film ou celui de la vidéo.

Nous ne donnons à notre sujet que des limites géographiques et chronologiques. Ces limites sont celles de la recherche de terrain effectuée ${ }^{1}$, ce qui ne préjuge pas de l'extension effective de la controverse. L'époque: nous remontons de l'époque actuelle aux années cinquante. Le lieu: la France, c'est-à-dire ceux qui, en France, prennent part
\end{abstract}


à ce débat, qu'ils soient ingénieurs, producteurs ou réalisateurs. Nous nous situons ainsi à l'intérieur d'un milieu, la télévision française et ses entours immédiats (journalistes et parlementaires spécialisés) $)^{2}$. À ces parties prenantes il faut ajouter sociologues et historiens du cinéma et de la télévision, puisque leurs commentaires sont parfois rétrospectivement utilisés par des acteurs du débat. Amené à reparcourir les positions des acteurs dans des controverses qui sont pour eux toujours actuelles, l'auteur a déjà fait plus d'une fois l'expérience des mobilisations inattendues. Ne voulant pas trancher, on se trouve mobilisable à volonté par le lecteur toujours désireux de nourrir la controverse qu'il s'agit, le temps d'une recherche, de suspendre.

La limitation au milieu de la télévision surprendra peut-être. Une controverse technique sur la production d'émissions en film ou en vidéo évoque irrésistiblement l'opposition plus connue et plus publique entre deux formes de spectacles, deux institutions et deux milieux: la télévision et le cinéma. Nous ne l'évoquerons pas directement. Non qu'elle soit négligeable, au contraire. Né dans les années cinquante, le conflit entre télévision et cinéma est aujourd'hui très profondément inscrit dans les mentalités des milieux professionnels, mais des profanes, simples spectateurs du grand et du petit écran, y interviennent: il s'agit donc d'une controverse complexe, jamais vraiment étudiée. Notre controverse technique et professionnelle, plus modeste, plus dissimulée, entretient bien sûr des rapports avec cette grande opposition. L'élucidation de ces rapports fait partie du travail entrepris ici.

Retour à la question inaugurale: quelle méthode de production choisir pour les émissions dramatiques: la vidéo ou le film? La question est posée à plusieurs époques. De quel droit dire que c'est la même controverse? Les outils techniques concernés ont évolué, les méthodes de production elles aussi. Les individus ont parfois changé de camp, les acteurs collectifs se sont transformés. Nous ne pouvons apporter maintenant qu'une réponse partielle: certains acteurs disent qu'il s'agit d'un conflit unique et ancien. Des professionnels de la télévision parlent de la controverse entre vidéo et film avec aisance, évoquant toute une histoire implicite, une tradition de controverse à laquelle on peut se référer par simple allusion, avec la certitude d'être compris.

Le lexique de la controverse présente un trait remarquable. On oppose en effet aussi bien « vidéo » à « film » qu'« image argentique » à « image électronique », « image chimique » à « image magnétique ». Le choix des termes recouvre des opérations sémantiques parfois complexes. Un exemple: le couple « image argentique », « image électronique ». On choisit une des caractéristiques techniques de l'outil pour désigner le tout (partie pour le tout, métonymie ou, pour être plus précis, synecdoque). De l'« image argentique » on passe aux outils qui permettent de produire cette image. Mais cette première métonymie conduit à une seconde: des outils on passe aux utilisateurs (les techniciens, les ingénieurs, tous les personnels « du film »), voire à tous les partisans supposés de l'outil. En même temps, si l'on évoque ces deux milieux, ces deux univers, en sélectionnant un élément technique, c'est bien parce que l'élément technique paraît ordonner l'univers, voire même le produire, qu'autour des outils des personnels sont déjà là, à leur poste, ralliés d'emblée, à la simple évocation de l'outil. Plutôt que stricte métonymie, il y a oscillation du tout à la partie, de la partie au tout. Les expressions choisies pour se référer à la controverse correspondent d'ailleurs à autant de manœuvres rhétoriques. Dire « vidéo », « film » (comme on l'a fait dans le titre de cet article), c'est choisir une métonymie durcie par le temps, se référer à des milieux autant qu'à des outils. À l'inverse, dire « image argentique », « image électronique », c'est inviter l'interlocuteur à contempler l'image et elle seulement, à se retirer de la société pour contempler les outils dans toute leur " pureté $»^{3}$. 
Nous avons restreint le lieu et l'époque, nous n'avons même plus, pour baptiser la controverse, de termes génériques. Pourtant l'objet est là, et la controverse persiste. Les efforts répétés pour la nier ou l'atténuer ont tous échoué. Il n'y a pas de conflit vidéo/film, disaient les ingénieurs des années soixante, aussitôt accusés par les réalisateurs de vouloir dicter leur choix, c'est-à-dire trancher dans la controverse. À l'occasion d'innovations techniques, certains acteurs annoncent aussi la fin prochaine de la controverse, sur un mode prophétique - ce qui a pour premier effet de la pérenniser, ne serait-ce qu'en la valorisant comme un moment long de l'histoire: « Le cycle TV/cinéma s'achève, nous avons maintenant un nouveau format. La technique électronique est l'héritière de ces deux expressions en n'en gardant que le meilleur $»^{4}$, dit ainsi un directeur de chaîne de télévision à propos de la Télévision à Haute Définition. Pour le profane, rappelons que la « Télévision à Haute Définition » (TVHD) est un terme-enveloppe (et un bon exemple de métonymie valorisante réussie) pour désigner un ensemble d'innovations techniques dont la caractéristique essentielle, à ce stade de nomination technique, est que nul ne peut les définir de façon précise en extension ou en compréhension. La TVHD en est, à l'heure actuelle, au stade expérimental.

Un cycle s'achève, un autre va commencer. Il n'est plus que d'attendre. Cette annonce prophétique de la fin de la controverse émane, typiquement, soit d'ingénieurs lassés de s'y trouver dénoncés, soit de réalisateurs et de personnels de production qui, comme on dit, « jouent la carte » du nouvel outil, c'est-à-dire cherchent à doter de « potentiel artistique » ce qui n'est encore qu'une machine, une « technique », mais pas encore " un mode d'expression »-pour employer leurs propres termes. Mais, par un effet de rémanence, cette controverse dont on annonce la fin repart bientôt de plus belle, alors qu'on la déclare privée de causes: le film a encore de beaux jours devant lui, ont ainsi répété certains réalisateurs à chaque « avancée » de la vidéo (dont la TVHD est le dernier avatar). Le prophète de la résolution du conflit par perfectionnement de la vidéo voit aussi son propos soumis à réinterprétation. Loin d'être un conciliateur, il est un partisan déguisé de la vidéo.

Étrange controverse. Les acteurs et les outils ont changé au cours du temps. Ceux qui en parlent soulignent, non sans ironie parfois, son ancienneté. Fausse controverse alors, à tout le moins conflit " mineur », " exagérément grossi » comme nous invitent à la considérer ceux des acteurs qui la minimisent ou annoncent sa résolution prochaine. Vraie controverse, répondent d'autres acteurs qui affirment son caractère irréductible, et dénoncent la mauvaise foi des conciliateurs apparents. La seule façon de trancher, non dans la controverse, mais sur sa nature, est d'en reparcourir, pas à pas, les différentes étapes. Enrichie par l'histoire, celle-ci a-t-elle été dénaturée? y a-t-il, dans le «vieux conflit entre image argentique et image électronique », des constantes?

\section{Aux origines de la télévision, une hybridation des outils sans conflits}

Si l'opposition entre film et vidéo existe ailleurs qu'en France, il faut noter que la période qui voit se constituer la télévision comme dispositif de laboratoire (1900-1945), avant que n'en commence l'exploitation industrielle, est aussi celle où les deux techniques télévision et film s'entremêlent. Si le cinéma a une définition ferme, à la fois comme 
outil, comme lieu géographique, comme pratique de divertissement - mais pas encore, ou très modestement, comme « art » spécifique - la télévision, quant à elle, n'existe pas. Son nom même ne sort du laboratoire que dans les années trente, et seuls le connaissent quelques passionnés, quelques curieux. Mais elle n’a ni " public », ni « professionnels ».

Le terme même utilisé pour définir l'invention a beaucoup varié, comme en témoignent les brevets recensés avec méticulosité dans l'histoire « juridico-technique » remarquablement précise d'Abramson (1987). En outre, les inventeurs travaillent à la fois sur la télévision, la photographie (elle aussi déjà constituée) et le film, et surtout ils mêlent dans leur travail beaucoup de ces outils, sans avoir le sentiment de marier des contraires. Il n'y a pas à choisir : à l'opposé, il faut mélanger. On n'invente pas, ou pas encore « la télévision », mais des dispositifs techniques de transmissions de l'image (fixe ou en mouvement) dont l'usage n'est pas défini. Ils serviront à transmettre des photographies, voire des films pour lesquels on envisage des dispositifs de développement très rapide afin de se rapprocher, par un léger " différé », de la transmission « en direct » ${ }^{5}$ d’images animées, réalisées d'abord en laboratoire puis à l'extérieur. Ils serviront à marier le téléphone et l'image (usage très tôt envisagé, avant la retransmission de spectacles). C'est le temps d'hybridations non conflictuelles entre l'image «argentique » et l'image «vidéo », que personne ne pense à opposer, pour la simple raison qu'elles n'existent pas encore, en tout cas pas dans ces termes.

\section{Controverse « préparatoire »: la dramatique en direct}

Par contre, l'époque de démarrage industriel et politique demande à être considérée plus longuement. Se constituent autour de l'outil vidéo, qui domine alors à la télévision, des groupes qui reprennent un héritage de l'avant-guerre (« les ingénieurs de la radiodiffusion », déjà désignés comme tels dès 1939) ou qui sont d'apparition plus récente (les « réalisateurs », dont le nom même émerge dans les années cinquante). Or il s'agit de deux collectifs qui vont jouer, dans les années suivantes, un rôle essentiel dans la controverse - ce futur n'étant porté par aucune tentation téléologique. Les choses auraient pu rester en l'état des années cinquante, en ce sens où les deux groupes, constitués, auraient pu continuer à s'affronter, à polémiquer, sans que cette opposition prenne forme technique, et que la technique devienne même, semble-t-il, le support majeur de l'opposition.

En effet, dans les années cinquante, il n'y a pas de controverse. La fiction réalisée par la télévision est entièrement tournée en vidéo. En vidéo, cela implique en studio et en direct. Les caméras sont très lourdes, et le réalisateur règne sur le studio dans une "régie » dotée d'une grande baie vitrée, d'où il donne les ordres aux cameramen, où il contrôle sur des « moniteurs » les différentes images, et où des techniciens vérifient la qualité du signal sur des écrans de contrôle. Le montage se fait en direct, le réalisateur choisissant sur les écrans l'image qui lui paraît la meilleure à un moment donné. Il s'agit alors d'une nouveauté dans l'usage de la caméra: sauf pour certaines scènes, le tournage d'un film de cinéma se fait à une seule caméra, plan par plan. La dramatique en direct crée autour d'elle un rituel de tournage qui emprunte beaucoup au théâtre: nombreuses répétitions, angoisse de la première, caractère éphémère de la représentation; auquel il faut ajouter le ballet de quatre caméras énormes, les pannes fréquentes - d'où un vocabulaire, pour parler de ces représentations, qui évoque parfois l'exploit sportif. 
Les réalisateurs qui pratiquent la dramatique (et qui deviendront assez vite, dans le commentaire critique qui entoure la télévision des années cinquante et du début des années soixante, « les » réalisateurs), ont une ambition, un modèle. Ils pensent au cinéma. Ils réalisent parfois des découpages par plan extrêmement serrés, qui rendent le tournage fort difficile sur le plan technique. Bref, ils se posent déjà, comme l'un d'eux l'écrit dans un livre de souvenirs en 1975, « les éternelles questions, comment faire semblant de faire du cinéma en faisant de la vidéo? » (Bluwai 1976 : 93). Simplement, ils ne savaient pas encore que la question serait «éternelle».

En face, « les » ingénieurs, du moins ceux que les problèmes de diffusion n'accaparent pas complètement (la mise en place du réseau de diffusion est l'opération cruciale) critiquent cette attitude. L'un d'entre eux, qui fut dès les premières années, et jusqu'à 1974, responsable de l'exploitation télévisée, chargé officiellement d'expliquer aux réalisateurs les problèmes techniques, porte un jugement global négatif, sans distinguer entre les époques, sur les réalisateurs qui n'avaient pas compris la « rationalité » des tournages vidéo, l'usage des plans longs et de la profondeur de champ qui permet d'éviter «le ballet des caméras », bref, ce qu'il appelle le « tournage à l'anglaise ». En effet, tourner en plans longs, en exploitant un seul angle, permet d'éviter les périlleuses commutations en direct d'une caméra à l'autre. De plus, se limiter à un seul angle, c'est aussi réduire les frais de décors.

Il y a des tensions sur les modalités d'usage de la vidéo, mais pas de controverse vidéo/ film, parce qu'il n'y a pas de film, ou très peu. À notre connaissance, une dramatique en $35 \mathrm{~mm}$ est tournée dans les années cinquante (une adaptation de Madame Bovary), dont le souvenir reste lourd chez les témoins des services techniques et administratifs comme l'un des premiers exemples de coûts élevés (alors que la télévision ne faisait pas de devis - mais, on le suppose, l'essentiel du matériel avait dû être emprunté ou loué à l'extérieur). L'on voit apparaître l'une des significations du couple vidéo/film. Vidéo est associé à production interne, "maison ", "nationale », et film à production externe, « privée ». Suivant que l'on valorise l'une ou l'autre, l'on sera conduit, au moins jusqu'en 1974, à valoriser le film ou la vidéo.

Cependant, cette dramatique vidéo qui essaie de ressembler au cinéma apparaît aussi comme un produit spécifique, et les réalisateurs de télévision, malgré leur envie de cinéma, peuvent camper sur une position originale. Les premiers critiques de télévision, à la fin des années cinquante, théorisent volontiers sur cet art nouveau. L'immédiateté de la transmission paraît garante d'un contact particulier avec le public. Un terme est souvent employé à propos du direct, celui de «magie »: « magie » du direct, du contact sans délai entre le spectacle et les spectateurs, comme au spectacle, mais avec des milliers de spectateurs. Cette valorisation de la dramatique en direct compense alors ce qui apparaîtra plus tard comme des défauts rédhibitoires. Les réalisateurs essaient de faire " comme du cinéma », mais ils font aussi autre chose, qui a sa "magie » propre. Quoique les milieux du cinéma regardent alors avec mépris la télévision, les réalisateurs de télévision peuvent espérer qu'un processus de reconnaissance artistique et théorique s'amorce - alors que ce processus est, pour le cinéma, en plein développement dans les Cahiers du Cinéma (côté critique) et dans la Revue internationale de Filmologie (côté universitaire) dont un essai classique d'Edgar Morin synthétise alors les travaux ${ }^{6}$. Au centre de cette théorisation, il y a l'idée qu'une technique de reproduction du réel a pu, dans la surprise, engendrer un art: ne pourrait-il pas en être de même pour la télévision, s'interrogent réalisateurs et critiques. 


\section{L'épanouissement de la controverse}

De 1960 à 1974 notre controverse connaît un âge d'or. Elle s'épanouit d'abord au sein du petit milieu professionnel, dans les années 1960-1964. Elle est reprise avec succès dans plusieurs revues spécialisées, revues critiques aujourd'hui disparues, presse professionnelle (Le Technicien du Film), dans les publications techniques (la revue technique de l'U.E.R, l'Union Européenne de Radiodiffusion, haut lieu de publication pour les ingénieurs), dans les souvenirs écrits ou oraux des témoins, et enfin, gage ultime de succès, dans la presse quotidienne et dans les rapports parlementaires.

À ce processus de publicité des débats est liée la grande variété des significations dont s'enrichit l'opposition vidéo/film. C'est alors que se constitue, pourrait-on dire, le répertoire de la controverse, où tous les participants postérieurs viendront puiser, supposant connue et simple une histoire complexe, expliquant le présent par le passé ou le passé par le présent sans répondre bien sûr à la question qui nous occupe: pourquoi cette réouverture perpétuelle d'un livre de querelles qu'en d'autres domaines on arrive à fermer puis à oublier définitivement?

La controverse se développe car les outils se diversifient. On peut désormais tourner en film. D'abord format amateur, le $16 \mathrm{~mm}$ est devenu format professionnel, et la fiction télévisuelle l'utilise de plus en plus à partir de 1958. En 1963, est mise au point une caméra légère $16 \mathrm{~mm}$ avec son synchrone sans film, baptisée la «Coutant », du nom d'un des ingénieurs qui la met au point. Cette caméra est destinée d'abord aux documentaires et aux reportages. Très vite, cependant, elle va être détournée et utilisée dans la réalisation d'émissions dramatiques. Dernière innovation majeure enfin, le magnétoscope est introduit en 1960. Il permet d'enregistrer ce qui était antérieurement réalisé en direct - sans aucune possibilité de montage, au moins au début. Il est donc possible de diffuser ces périlleuses dramatiques vidéo en différé.

Malgré cette diversité de choix, les acteurs continuent de proposer de la controverse une version binaire: vidéo ou film. Une version ou plutôt des versions, car l'opposition n'est pas constituée de la même façon par les différents témoins. Soit, pour la période 1960-1964, un ingénieur:

« Il y avait une grosse bagarre à l'époque. La direction des programmes [...] était très favorable à ce qu'elle appelait le filmage. Les réalisateurs venaient du cinéma, d'où un phénomène de rejet de la vidéo. En face, les spécialistes techniques et les ingénieurs étaient formés par la télévision ».

Lingénieur qui parle est celui, responsable des moyens film, qui mesure tout l'attrait qu'exerce alors sur les réalisateurs l'outil film. Selon ses propres termes, il essaie « d'imposer le $16 \mathrm{~mm}$, dans un milieu qui ne jure que par le $35 \mathrm{~mm} »$.

Chez les dirigeants de la télévision d'alors (quelle que soit leur formation d'origine), on favorise très vite le film. Le directeur de la deuxième chaîne (1964-1967), mémorialiste passionné d'un moment qu'il n’a pas vécu, et panégyriste d’un de ces prédécesseurs disparu en 1964, écrit dans un livre publié peu de temps après son départ:

«Stellio Lorenzi (réalisateur fameux d'alors) et quelques réalisateurs, les techniciens ou plutôt leurs chefs, quelque critique pontifiant, chantent à longueur de journée les vertus du direct [...]. Malgré leur camarilla, Albert Ollivier - directeur des programmes de 1960 à 1964 - impose le développement des moyens film ». 
Une telle déclaration oppose les dirigeants (sensés, lucides, rationnels) et partisans du film, aux personnels (" quelques » réalisateurs, les techniciens « ou plutôt leurs chefs »), partisans de la vidéo.

\section{Des controverses emboîtées}

Nous voici dans un maquis de sous-controverses: $16 \mathrm{~mm}$ ou $35 \mathrm{~mm}$, magnétoscope ou direct, direct ou $16 \mathrm{~mm}$ (sans compter la pratique des inserts filmés qu'on intègre dans une dramatique en direct - aux alentours de 1960). De surcroît, les schémas de mobilisation diffèrent selon les interlocuteurs. Les réalisateurs sont favorables au film dans le premier témoignage. Certains réalisateurs sont opposés au film dans le second, et « chantent les vertus du direct». Essayons d'y voir plus clair.

D'abord, le débat entre vidéo en direct ou en différé ne survit pas longtemps à l'arrivée du magnétoscope. Les défenseurs de la dramatique en direct, certes, adoptent vite le ton de la nostalgie, pour regretter le «climat » du tournage, pour évoquer 《le sentiment d'être seul maître à bord après Dieu » ${ }^{7}$ que donne le tournage d'une dramatique en direct: aspect politique d'un choix technique. La dramatique suppose, le temps des répétitions et du tournage, une soumission totale d'un personnel nombreux derrière le réalisateur dont tout dépend, puisque la diffusion est en direct.

Mais cet attachement à la fois politique et esthétique ne résiste pas à l'isolement. La « magie du tournage » spécifique de la dramatique vidéo, cette tension « unique » qu'apportait la diffusion en direct, n'est, pour l'essentiel plus défendue par la critique, et, déclare-t-on, pas perçue par «le public»-alors que les incidents multiples, les ombres de la perche qui soutient le micro, font les délices ironiques des commentateurs du dedans et du dehors. La fiction télévisuelle perd sa «magie » spécifique, et une théorie esthétique qui pouvait la faire rivaliser avec la fiction cinématographique s'effondre. Les réalisateurs, minoritaires, qui défendent la dramatique en direct cèdent très vite.

Les dramatiques en studio seront donc tournées sur magnétoscope : c'est le choix de certains ingénieurs. Le magnétoscope, lancé par la firme Ampex aux États-Unis en 1955, arrive en Europe en 1960. C'est un ingénieur de l'O.R.T.F, personnage essentiel car gourmand d'innovations techniques, qui fait le voyage en Grande-Bretagne pour le voir le premier. Poussé par les ingénieurs responsables de l'exploitation télévisée (les rapports des services techniques citent comme un progrès essentiel l'accroissement du nombre d'heures), le magnétoscope est rapidement utilisé, non seulement pour les retransmissions d'événements, mais aussi pour les émissions dramatiques.

Le magnétoscope est pour les ingénieurs l'outil idéal de la production télévisée. Il permet de sauver la production dramatique vidéo menacée par les périls du direct. Les ingénieurs ont fait construire, depuis le milieu des années cinquante, de grands studios vidéo - opération qui fera l'objet, de la part des parlementaires relayés par des journalistes spécialisés ${ }^{8}$, de vives critiques car jugée trop coûteuse. Les calculs des ingénieurs, qui sont alors en position de gérer la production télévisée, démontrent pourtant qu'elle est moins chère qu'une dramatique film, sous la réserve essentielle que les studios soient occupés, et que certaines modalités d'usage soient respectées. 
Car une autre controverse naît à propos du magnétoscope. Les ingénieurs favorisent des cadences plus rapides, un tournage en continuité complet, « dans les conditions du direct » (éclairage sous de multiples angles, montage par commutation entre caméras). Cet argument ne tient que si les cadences nécessaires sont tenues par les réalisateurs. Mais ils ne jouent pas ce jeu, et comme ils l'ont fait avec le direct, essayent de faire « du cinéma avec de la vidéo ». Ils ne tournent pas d'une seule traite, mais en séquences longues et essayent de faire pratiquer un montage très délicat car non prévu par les fabricants à l'origine, aux ciseaux, à la colle et au microscope - travail manuel « artisanal » qui, à l'opposé de l'univers électronique, rappelle le montage film. À partir de 1966, l'arrivée du montage électronique, sans cesse perfectionné depuis, permet une résolution partielle de la controverse, qui se déplace désormais vers la durée du montage magnétoscope - coûteux, à réduire au minimum pour les uns, indispensable au travail artistique pour les autres.

Même avec le magnétoscope, avec une ébauche de montage, la dramatique vidéo continue de faire l'objet de vives critiques. Celle plus souvent citée concerne la lumière. En film, on le sait, on tourne à une seule caméra, ce qui donne au directeur de la photographie (fonction professionnelle immédiatement en dessous du metteur en scène dans la hiérarchie des métiers) des possibilités d'éclairage très importantes. Ce travail de la lumière, disent tout à la fois les théoriciens du cinéma, les professionnels, les critiques, est une partie essentielle de « l'art cinématographique ». Sur un plateau de vidéo, nous dit un réalisateur faisant a posteriori le récit de la « crise de la dramatique vidéo »:

«Quels que soient les efforts des directeurs de la photographie - et ceux de la télévision sont parmi les meilleurs - [...], la multiplicité des angles simultanés de prise de vues entraîne un éclairage qui, à peu de chose près, est celui du scialytique d'une salle d'opération. D'où cette impression de lumière neutre - où beaucoup d'angles sont d'ailleurs sacrifiés - qui est si caractéristique des émissions dramatiques vidéo et qui, la pauvreté des décors aidant, les fait reconnaître à des lieux ».

Oubliée, la magie du tournage en direct; reste la lumière qui n'agit pas seule (cf.: « la pauvreté des décors aidant ») et qui est autant un défaut spécifique qu'une marque indélébile qui «fait reconnaître » la technique. Comme les humains, les objets techniques portent parfois des stigmates ${ }^{9}$.

Le cinéphile amoureux de la lumière peut se reconnaître ici dans cette (auto) critique du réalisateur de télévision, qui s’accompagne volontiers d'une dénonciation rituelle des techniciens et des ingénieurs, partisans de la vidéo, indifférents à l'art. Pourtant l'attachement des ingénieurs pour la dramatique vidéo n'est pas que froid souci d'économie. Il a aussi, indissociablement, un aspect sentimental, certes plus confidentiel. L'ingénieur de la télévision souvent se reconnaît dans la technique vidéo, et éprouve même quelques répugnances envers les techniques du cinéma, au moins à l'époque qui nous occupe. Tel l'auteur de cette phrase extraite d'un volumineux rapport célébrant « l'évolution des techniques O.R.T.F. » et rédigé par des ingénieurs de "la Maison »: « la filière de production film est un véritable maquis et l'ingénieur s'y sent très mal à l'aise ». "L'ingénieur »: à nouveau un groupe tout entier est mobilisé « contre » le film. Pourtant, des ingénieurs, parfois contraints, prennent le parti du film: la pression est trop forte. 


\section{Le film $16 \mathrm{~mm}$ « compromis idéal »}

Magie du direct enfuie, soutien critique disparu, éclairage chirurgical: ces condamnations successives de la dramatique vidéo expliquent pourquoi les réalisateurs, alors que les tournages en film sont possibles, se ruent sur les caméras disponibles, et notamment sur la caméra $16 \mathrm{~mm}$ légère, la «Coutant », outil qui provoque toujours une forte nostalgie dans les milieux professionnels, où il est resté légendaire. Outil sans stigmates, à l'inverse de la technique vidéo. Certes, le $35 \mathrm{~mm}$ du cinéma reste la technique suprême: mais la pédagogie des ingénieurs, et surtout le chômage croissant des réalisateurs, ont fini par porter. À défaut du $35 \mathrm{~mm}$, idéal inaccessible, on préférera le $16 \mathrm{~mm}$, « compromis idéal », selon le mot de plusieurs réalisateurs. Conçue initialement pour le reportage et le documentaire par une firme extérieure à la télévision, la Coutant va être détournée et utilisée par les réalisateurs des émissions dramatiques ${ }^{10}$.

Ces réalisateurs partisans du film trouvent un soutien en la personne des dirigeants de la télévision, qui dramatisent volontiers le « combat » en faveur du film, comme nous l'avons vu. Mais l'immense majorité des réalisateurs a essayé, dès que cela a été possible, de faire du film. Les dirigeants ont été à leur côté, pour des raisons à la fois d'esthétique et de politique. Les raisons politiques sont rarement mises en avant. Le film suppose le plus souvent le recours à la production extérieure, c'est-à-dire à des personnels plus faciles à gérer que les techniciens « au statut » et prompts à la grève, ou même que les ingénieurs qui ont sur l'outil et la gestion beaucoup (trop) d'idées.

Parallèlement, la nostalgie du cinéma qui s'épanouit chez les réalisateurs se retrouve chez les dirigeants. Dans ces années-là, « la télévision » esquisse un rapprochement avec « le cinéma », c'est-à-dire que certains dirigeants se préoccupent de faire venir à la télévision des « grands metteurs en scène » du cinéma, et aussi de réussir à diffuser à la télévision un nombre plus important de films. Le film, supposé « attrayant pour le spectateur », a aussi pour lui ce statut d'art qui fait défaut à la télévision. Diffuser

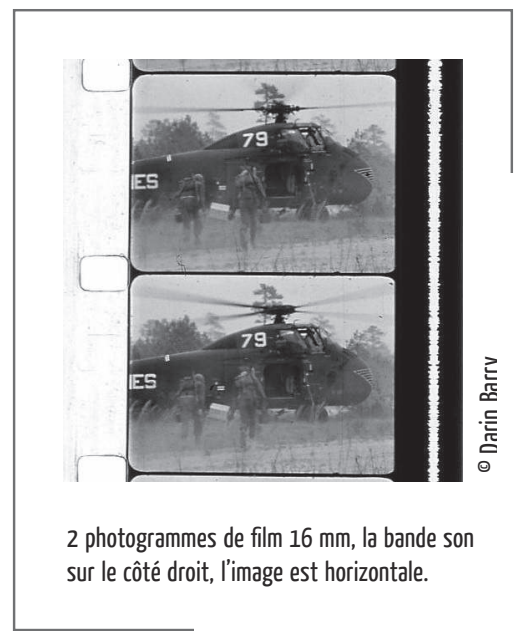


des films, faire venir des metteurs en scène, et... travailler sur pellicule - tout cela, c'est « se rapprocher du cinéma »; à nouveau l'image est envahie d'objets « extérieurs ».

Trois groupes jouent un rôle essentiel à ce moment de la controverse, et identifient partiellement leur destin et la constitution de leur identité à des objets ${ }^{11}$. Les ingénieurs s'associent le plus étroitement à la vidéo, qu'il s'agisse de son utilisation pour le direct puis pour le différé. C'est pourquoi, dans la période qui va suivre, leur déclin est indissociable du déclin de cette technique. Les réalisateurs vont d'un outil à l'autre, d'une modalité d'usage à une autre, avant de privilégier le film $16 \mathrm{~mm}$. Les dirigeants, enfin, fondent partiellement la reconnaissance d'une compétence télévisuelle sur le développement de la fiction film. En cela ils sont les alliés des réalisateurs.

Les acteurs eux-mêmes ont trouvé pour se définir des termes qui associent les groupes aux outils et aux modalités d'usage de ces outils. Les années soixante et soixante-dix ont connu, à la télévision, une opposition, parfois encore utilisée, entre « saltimbanques » et " géomètres » qui implique deux façons de considérer les outils, l'une sur le mode de l'art, de la fantaisie, de la liberté, l'autre sur le mode de la rigueur, de la mesure. Les géomètres ont d'abord été les techniciens responsables des équipements vidéo et les ingénieurs, soucieux d'une pleine utilisation des studios vidéo et, tout particulièrement, indifférents aux questions de l'éclairage. La mesure du géomètre, c'est à la fois celle, trop grossière, du signal technique et celle, trop sévère, du plan de travail et du devis.

\section{La vidéo condamnée, ou pourquoi une « technique » n'est pas nécessairement un « mode d'expression »}

À partir de 1970, les ingénieurs s'engagent dans un combat visant à rentabiliser l'outil de production vidéo ; ils sont secondés (partiellement) par des administrateurs venus de la haute fonction publique dont l'influence ne cesse de croître à la télévision: les réalisateurs les rebaptisent très vite... géomètres. La télévision fait l'objet, à partir de 1971, de multiples plans de réorganisation. La production télévisée est désormais structurée en « régies » ordonnées autour de trois moyens techniques: vidéo fixe, vidéo mobile, film (nous reprenons ici l'ordre « officiel » de présentation).

Un mot d'explication sur la «vidéo mobile». Nous n'avons parlé que de la dramatique, et seulement de la dramatique en studio. N'imaginons pas que la vidéo ait été tout entière cantonnée au studio. Depuis les années cinquante, des caméras vidéo sortent des studios. Mais il s'agit d'une opération technique lourde et difficile, qui suppose des cars vidéo énormes, et, le plus souvent des émetteurs en direct. C'est en effet à la transmission d'événements en direct que la «vidéo mobile» est utilisée.

La « vidéo mobile», si elle sert à l'enregistrement de débats et de variétés, est aussi prévue pour la réalisation de tournages en vidéo. Dès 1966, les publications des services techniques font état d'« expériences » de tournages avec le car vidéo mobile. Dans un document officiel datant de 1973, l'ingénieur auteur du chapitre sur les moyens techniques nous montre des outils égaux, cohabitant harmonieusement et sans conflits: "vidéo fixe, vidéo mobile, film, mieux que trois techniques: trois moyens d'expression de la télévision ». À nouveau, il plaide pour l'égale dignité des 
techniques. Mais, aux yeux des réalisateurs, le film reste bien le « moyen d'expression » majeur, les autres techniques demeurant, pourrait-on dire, de simples outils.

Car la vidéo mobile n'est pratiquement pas utilisée comme outil dramatique. Quant à la vidéo fixe, ses problèmes sont résumés dans une formule fréquemment employée alors, la « crise de la dramatique vidéo fixe ». La vidéo fixe, outil des émissions de débats, variétés, jeux, ne convient pas aux émissions dramatiques: c'est ce qu'écrivent, de plus en plus souvent, critiques, journalistes, réalisateurs. «La vidéo fixe est-elle condamnée? », s'interroge telle publication de la Société française de production, héritière de l'O.R.T.F., en 1976. Et d'énoncer, puisant largement dans le répertoire déjà constitué de la controverse, les caractéristiques qui condamnent la vidéo fixe: l'éclairage, les progrès du film en extérieur, etc.

Bref, la condamnation peut s'expliquer par des motifs " purement techniques ». C'est une comparaison entre pays qui nous permettra de défaire momentanément cette argumentation. À l'époque où la France s'interroge, dans ce milieu des années soixante-dix, sur l'opportunité de continuer à tourner des dramatiques vidéo en studio, les télévisions américaines et britanniques enregistrent en continuité quantité d'émissions dramatiques de ce type, à des cadences extrêmement rapides. Ce sont les « comédies de situation », que la France va importer plus tard, mais qu'elle ignore alors. Le débat sur la technique est un débat sur les modalités d'usage qui paraissaient possibles à un moment donné. Il dit aussi, à une époque où ceux-ci estiment leur pouvoir considérablement entamé, le poids relatif des réalisateurs de télévision qui ont été les premiers généraux, mais aussi, dès que la possibilité s'est présentée, les premiers déserteurs de ces studios de vidéo.

À nouveau, le conflit « technique » est un conflit sur les modalités d'usage. Selon un historique de la vidéo publié en 1974 dans un rapport parlementaire (Chinaud 1974 : 77), la " production de création » a obtenu des tournages vidéo en séquence, et le recours au montage (devenu entre-temps montage électronique). D’où un allongement de la durée du travail - puisque, de nouveau, les réalisateurs « se rapprochent du cinéma » : préparation et montage longs, tournages par séquence, changements de décors et de costumes. Mais, poursuit le même rapport, « des limites doivent y être apportées afin que soit maintenu un juste milieu entre la nécessité de rigueur de la prise de vues en direct et la liberté d'improvisation du tournage film, et que la souplesse ne conduise pas à la facilité ». Souplesse (la vidéo ressemble un peu au film), facilité et laxisme (si elle veut y ressembler trop, elle coûte trop cher), on a reconnu là une figure déjà familière de notre répertoire.

\section{Avatars de la vidéo, compromis manqués}

Cette crise dans l'utilisation des moyens vidéo appelle d'autres réponses, plus confidentielles que des plans de réorganisation. Dans les laboratoires, des ingénieurs et des techniciens travaillent à la transformation de la vidéo. Une première idée apparaît au début des années soixante: entre les exigences « cinématographiques » des réalisateurs et les exigences « télévisuelles» des responsables techniques; le compromis le plus simple n'est-il pas de réaliser un outil qui incorpore des caractéristiques de l'un et de l'autre? Ce monstre technique, c'est la vidéo-film, exemplaire 
échec technique. Le procédé consiste à mettre en studio trois caméras de film. Le réalisateur a accès simultanément aux trois images, sur des écrans de contrôle. Il doit, en principe, tourner en direct ou par larges séquences, en commutant les caméras. Le moteur correspondant se met alors en marche, et la précieuse pellicule film est impressionnée. Le procédé est utilisé vers 1960, abandonné puis repris en 1967 pour la couleur (la télévision en couleur a été officiellement lancée le ler octobre 1967), puis abandonné définitivement.

Pourquoi cet abandon? Les réalisateurs qui utilisent l'outil ne le font pas conformément à l'usage souhaité. Soit ils essayent de retarder jusqu'au dernier moment le choix de l'image, c'est-à-dire tournent avec les trois caméras simultanément, ce qui entraîne une consommation élevée en pellicule et la réapparition du montage dont l'élimination était un des objectifs clefs des inventeurs du dispositif. Soit ils abandonnent les trois caméras pour ne tourner qu'avec une seule, c'est-à-dire pour faire du cinéma, pour retrouver les possibilités d'éclairage. L'outil est d'emblée vidé de tout son sens. Le compromis proposé ne fonctionne pas.

Pour séduire les « saltimbanques », les laboratoires de la télévision travaillent dans une autre direction. Le montage magnétoscope a fait des progrès considérables. Les caméras vidéo n'ont cessé de se perfectionner. Les réalisateurs se sont passionnés pour les tournages en extérieur et le film léger. Film est devenu synonyme de légèreté. De.la vidéo mobile (mobile mais lourde), on va donc passer à la vidéo légère, et, une nouvelle fois, essayer de transformer la vidéo en film, en ne tournant qu'à deux caméras ou à une seule.

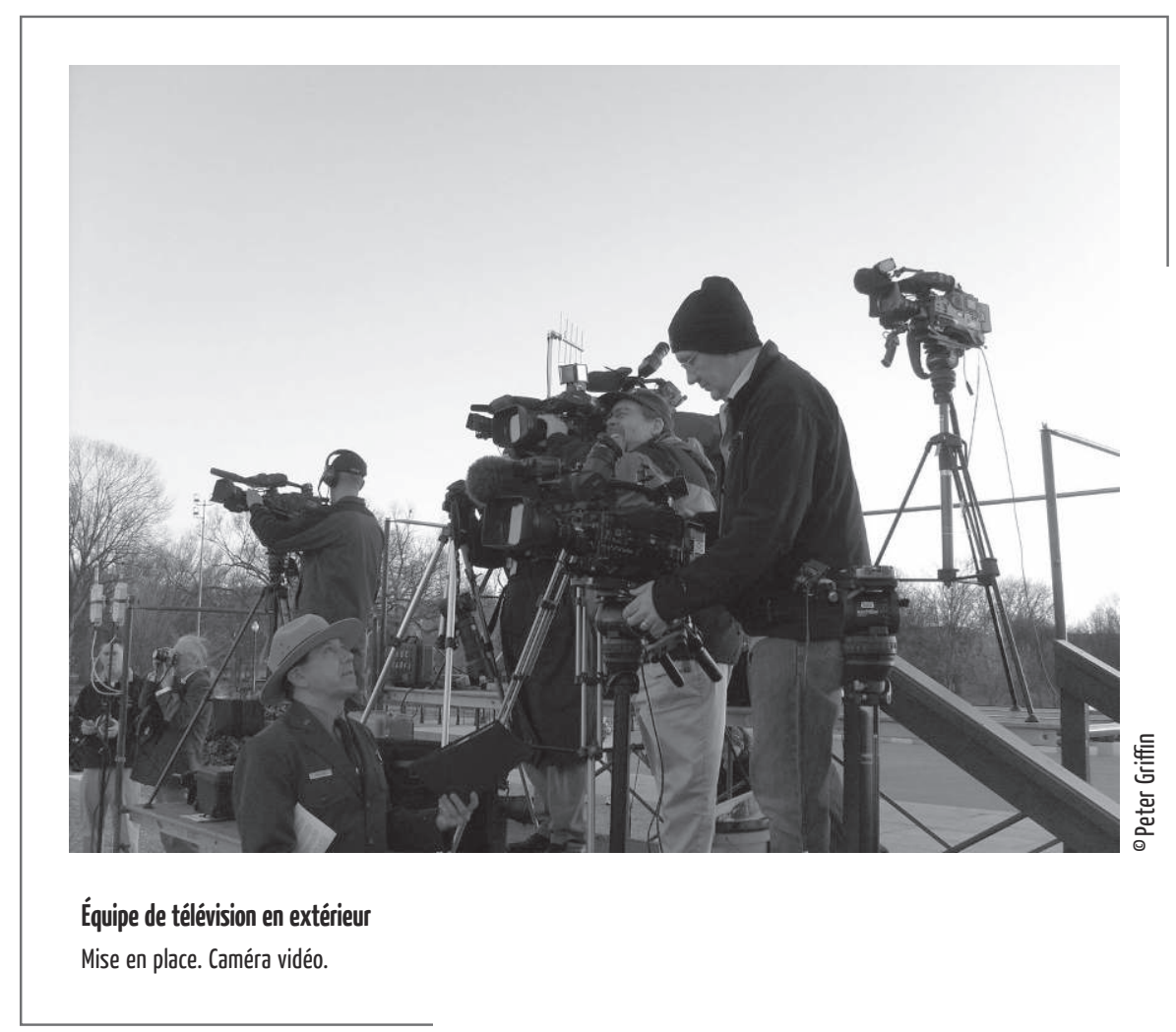


Comme pour le film de cinéma, les progrès de la vidéo légère n'ont pas pour origine première la satisfaction des réalisateurs d'émissions dramatiques. C'est dans les milieux du reportage en direct, chez des techniciens proches des journalistes, que s'accomplissent les progrès décisifs qui rendent disponibles, à partir de 1976, des caméras vidéo légères et des magnétoscopes portables. Le car vidéo, énorme machine qui avait vu sa taille se réduire au milieu des années soixante, grandir à nouveau au moment de la couleur, n'est plus seulement réservé aux grandes occasions, aux grands directs techniques. Il devient un outil de reportage.

Dans le même temps, les services techniques proposent à des réalisateurs de tourner des fictions en vidéo « mono-caméra » ou « bi-caméra ». Légèreté du cinéma, possibilités d'éclairages plus subtils que le studio, plusieurs expériences sont faites en ce sens. En 1971, un car «mono-caméra » est réalisé et utilisé dans quelques expériences de tournage dramatique. Les possibilités de montage paraissent trop limitées et la caméra demande un long travail de mise au point. La tentative est un nouvel échec. Mis en service en juin 1973, un car «bi-caméra » permet un tournage par courtes séquences - et non plus plan par plan selon le modèle cinématographique strict. Le mouvement se poursuit. En 1977-1978, la Société française de production ainsi que des sociétés privées s'équipent de studios prévus. Pour le tournage vidéo mono et bi-caméra. Mais dans le studio, bien souvent, les choses ne se passent pas « comme elles devraient ». Une des deux caméras reste sur le sol, et le compromis échoue de nouveau.

Aujourd'hui, le débat court toujours. Dans un genre télévisuel nouveau, les « comédies de situation ", le tournage a lieu en studio multicaméra: la «dramatique vidéo fixe» est donc revenue. Mais il s'agit d'un genre méprisé par les réalisateurs, sans qu'on sache si c'est la technique qui est méprisable ou le contenu que l'on choisit d'enregistrer à l'aide de cette technique. Pour les tournages en extérieurs, pour les séries de fiction les plus prestigieuses, le film domine toujours sur le plan français et international. Parti enquêter auprès des différents milieux, un journaliste conclut: « quelle que soit l'évolution de la technologie, la question fondamentale du choix de la technique de tournage demeure $»^{12}$.

\section{Retour à la question}

Alors, controverse vraie ou fausse? Notre récit peut être résumé ainsi. Autour d'une controverse permanente, liée à « l'éternelle » question des réalisateurs (comment faire semblant de faire du cinéma avec de la vidéo) se sont greffés une série de conflits périphériques, qui ont compliqué et enrichi la controverse initiale. Premier conflit périphérique : avec des stratégies différentes, film et vidéo ont servi de support pour la constitution (manquée en tout ou en partie, pour des raisons qui ne tiennent pas seulement à la technique) des groupes professionnels, ingénieurs, réalisateurs, et, dans une moindre mesure, dirigeants. Aux techniques film et vidéo se sont aussi associés d'autres débats politiques et économiques, avec des jeux d'alliances complexes. Aux débuts de l'histoire de la télévision, le film est associé aux tournages en extérieur, à la lourdeur du $35 \mathrm{~mm}$ et du montage (puisqu'il n'y a pas de montage vidéo). Aux 
débuts des années soixante, le film $16 \mathrm{~mm}$ devient synonyme de légèreté et se pare de séductions nouvelles. Malgré les luttes des ingénieurs de la télévision, la vidéo incarne la lourdeur, les coûts excessifs, voire le « corporatisme » de la télévision.

Tout se passe comme si les évolutions techniques de la télévision étaient partiellement commandées par la question suivante: comment séduire le réalisateur de fiction? Comment lui offrir quelque chose qui obéisse à des normes de télévision (essentiellement des contraintes de rentabilité et de temps), mais qui ressemble au cinéma. C'est ainsi que l'on peut analyser certaines des transformations techniques de l'un et l'autre support, comme recherches du bon compromis. Le film est cher et lourd: on offrira du matériel léger acceptable pour des raisons économiques, mais quand même du film. Pour tourner en studio, vite (comme le souhaitent les ingénieurs) mais sur pellicule, comme au cinéma, on invente la vidéo film (mais on oublie un élément essentiel, l'éclairage). Échec. Pour rendre à nouveau la vidéo acceptable, on la rend légère, puisque le tournage léger en extérieurs a triomphé au cinéma comme à la télévision: c'est la vidéo légère, voire même, rattrapant les possibilités d'éclairage, la vidéo mono caméra. Très souvent les techniques utilisées ne sont pas conçues au départ pour les émissions de fiction: elles viennent du journalisme et du reportage, milieu professionnel et d'un genre télévisuel relativement isolés.

Les difficultés de ces compromis tiennent pour beaucoup dans les réactions de ces réalisateurs. Au détournement de technologies mises au point dans les laboratoires (outils du reportage utilisés pour la fiction), ils en ajoutent d'autres et poussent toujours un peu trop loin la ressemblance avec le cinéma: ils abandonnent des caméras pour tourner plan par plan ou séquence par séquence et retrouver les possibilités d'éclairage. Ils s'obstinent à monter alors que cela leur est déconseillé ou interdit. Ils allongent la durée des plans de tournage et de montage. En fait de compromis, on en revient à des tournages cinéma classiques, mais en format $16 \mathrm{~mm}$. Quand toute possibilité de reconnaissance artistique du média disparaît, ce désir de film (support technique) à l'intérieur de la télévision devient, de façon définitive, une façon de réaliser son désir de cinéma (mode d'expression). Le support film permet ainsi de compenser, à l'intérieur même de la télévision, le défaut de reconnaissance artistique du média ${ }^{13}$.

\section{Images pures et impures, techniques stables et instables}

Arrêtons-nous un instant sur ce désir de cinéma qui a relancé plusieurs fois notre controverse. Donner à la télévision la dignité du cinéma en recourant à des transformations techniques: aurions-nous dit le fin mot de la controverse, à défaut d'y mettre fin? C'est en tout cas une invitation que nous lancent les critiques du cinéma quand ils regardent la télévision. À l'écran, sur le petit écran même, la différence entre la fiction sur vidéo et la fiction sur film se voient. Comparant trois soap operas (feuilletons de l'après-midi) tournés en vidéo et une série, Dallas, tournée en film, Les Cahiers du Cinéma concluent: « Après cette débauche de vidéo-fiction [...] le moindre plan de Dallas semble follement romanesque: décidément, la matière cinématographique garde quelque chose de la photo fixe, dont elle est issue $»^{14}$. 
Voilà qui paraît apporter de l'eau au moulin des réalisateurs et à leur volonté de tourner en restant près du cinéma puisque, au moins pour un œil exercé, ça se voit. Qui plus est, l'évocation de la photographie nous renvoie à des oppositions très anciennes, liées à la «nature » même des images. Nous savons pourtant maintenant que la « magie » de l'image film et la «pauvreté » de l'image vidéo sont le résultat d'un long processus. Citons un autre point de vue qui montre les limites des comparaisons « purement techniques ». En 1974, un rapport parlementaire s'exerce à départager ainsi les deux procédés de tournage, avant de concéder: «sur le plan de la qualité technique, la comparaison entre vidéo et film est extrêmement difficile [...]. Pour quelques paramètres particuliers, l'un ou l'autre des procédés offre des avantages indéniables » (Chinaud 1974 : 72). Mais qu'on glose un peu plus loin, et le fond se dérobe. Un exemple: "C'est que, sauf anomalie de fonctionnement (sic, mais l'anomalie serait immédiatement réintroduite dans la controverse par un partisan du film), l'image vidéo est d'une fixité très supérieure à l'image film (absence de mécanismes d'entraînement) et qu'elle est exempte de rayures et de poussières ». On peut ainsi continuer d'additionner des "paramètres particuliers ", des critères d'appréciation qui n'ont que le défaut de l'incommensurabilité. Le conflit «technique », qu'on espère clair parce que discutable sur un plan «purement technique » se dissout, redevient un conflit à de multiples dimensions, durablement insoluble.

Nous irons un peu plus loin que cet acteur. Plus iconoclaste qu'iconophile, nous avons du mal à vénérer l'image seule. Une considération patiente de l'histoire fait se mêler à ce " grain plus fin », à ces purs mérites du film, tant d'autres choses, des traditions, des habitudes, des rapports physiques avec les outils, des rapports avec les genres télévisuels mis en œuvre ${ }^{15}$. Les métiers du cinéma (à l'inverse de ceux de la télévision) réussissent à communier dans " l'amour de l'art », c'est-à-dire l'amour de « l'image de cinéma » pure et inchangée, amour nourri désormais largement par la haine des images commerciales de la télévision (Darré \& Heinich 1987).

Or, la stabilité de l'image cinématographique, c'est pour beaucoup celle des outils qui permettent de le produire: ce critère permet d'opposer autant les deux techniques de production que les deux milieux. Malgré des évolutions magnifiées notamment par ceux qui souhaitent le rapprochement entre cinéma et télévision, malgré la mise en valeur des « trucages » ou des «nouvelles images » (la deuxième expression est bien souvent un nouveau baptême pour des procédés anciens), le cinéma se fonde largement sur un ensemble de techniques simples, vite assimilées, partageables et donc très vite oubliées, ou bien, si l'on préfère, «mises au service » des ambitions artistiques. Le cinéma est le lieu d'un véritable déni de sa technicité, à quelques exceptions près.

Cette observation d'un réalisateur de télévision qui a travaillé en film résume assez bien à la fois ce déni et cette stabilité de la technique: "un des avantages du cinéma dont nous n'avons pas parlé, c'est de démarrer discrètement, presque incognito ». Mais cet incognito est le résultat d'une longue conquête: d'abord d'une stabilisation des outils techniques, dont l'histoire reste profondément mal connue. Tout se passe comme si le cinéma n'avait que des artistes, à la rigueur des techniciens, mais pas d'ingénieurs, pas de concepteurs, sauf aux origines. Ensuite, ils s'effacent, modestes, des dictionnaires, des histoires du cinéma. À l'inverse, en télévision, les ingénieurs (à l'intérieur ou à l'extérieur de la télévision) ont joué un rôle essentiel, et les innovations techniques de la vidéo n’ont pas cessé. Les techniques de la vidéo sont comme obsédées par le changement ${ }^{16}$. 
Il y a là peut-être une ultime explication à la pérennité de notre controverse. Tout se passe comme si les professionnels de la télévision avaient utilisé la référence à une controverse vidéo/film pour se doter d'une tradition bien particulière, pour réordonner une longue suite de conflits que le cinéma voile dans un amour indifférencié de sa technique et de son art. À défaut d'avoir stabilisé leurs outils, les professionnels de la télévision ont inscrit au cœur de leur histoire une « grande » controverse qui a réussi à en absorber quantité d'autres plus petites, à relier entre eux des acteurs et des objets de grandeur très différente ${ }^{17}$. Cette controverse est aussi un travail de la mémoire qui n'est pas sans évoquer un processus bien connu des historiens: comme on annexe à son pays rétrospectivement des territoires, l'on se dote de précurseurs, l'on rallie au présent des outils et des hommes du passé (Beaune 1985). 
1. Ce texte s'appuie sur plusieurs travaux antérieurs, réexploités ici dans une perspective nouvelle. Il s'agit principalement d'une étude pour le Ministère de la Recherche (Bourdon et Despratx 1988), et d'une thèse soutenue en 1989 et publiée depuis (Bourdon 1990). De son chapitre IV sont extraites toutes les citations dont les références ne sont pas précisées ici.

2. Les groupes impliqués dans la controverse sont peu nombreux. Parlementaires et journalistes ne sont que quelques individus très influents. Les professionnels de la télévision concernés sont une centaine de personnes au début des années soixante, peut-être trois fois plus nombreux à la fin des années soixante-dix, essentiellement à cause de l'accroissement du nombre de réalisateurs de télévision.

3. Les controverses techniques sont remplies de ces ligures de styles plus ou moins figées, qui correspondent à autant de manœuvres des acteurs (choix d'un trait valorisant de l'outil, mobilisation d'alliés autour de l'outil, etc.).

4. Cité par Thomas et Méadel (1992).

5. Les termes « différé » et « direct » sont bien sûr anachroniques, et utilisés par simple commodité. Ce vocabulaire-là n'est pas non plus fixé aux origines.

6. Morin (1956), tout entier consacré à l'élucidation anthropologique de la «magie » du spectacle cinématographique, contribuant par là même à conforter le statut social de son objet d'étude.

7. C'est ce qu'écrit un réalisateur dans Le Technicien du Film en 1958.

8. Un exemple de cette forte connivence entre journalistes et parlementaires spécialisés est donnée par Durieux (1972), qui contient un chapitre lourd de critiques sur les «techniciens »-c'est ainsi que sont désignés les ingénieurs.
9. Sur l'étude sociale du stigmate appliqué au sujet humain, voir Goffman (1975).

10. La genèse de cet outil capital dans l'histoire du cinéma et de la télévision ne nous est pas connue.

11. Sur les rapports entre professionnels et outils techniques. Cf. Latour (1989: 236-258).

12. Sortant du cadre français, j'extrais cette citation du magazine professionnel britannique Broadcast ( $1^{\text {er }}$ mars 1991).

13. Sur le désir de cinéma - le film long métrage -, présent chez tous les réalisateurs de l'audiovisuel et au principe de leur carrière, voir Thonon (1991).

14. Michel Chion, "Une journée au soap opera », Les Cahiers du Cinéma 72, avril 1987.

15. Consacré à la seule controverse vidéo/film, cet article n'entend pas recenser tous les liens qu'ont tissés autour d'eux ces procédés techniques. Outre Morin déjà cité, consulter Metz (1977), sur la position du spectateur, et Perriault, sur les ancêtres techniques du cinéma (1978).

16. Paradoxe: c'est partiellement le désir d'être stable comme le cinéma qui a rendu les techniques vidéo instables dans leur poursuite d'une illusoire ressemblance avec le cinéma.

17. Au sens que donnent à ce terme Boltanski et Thévenot (1987), même si je n'ai pas cherché systématiquement à appliquer les notions qu'ils développent. Lopposition entre nature industrielle et nature de l'inspiration, notamment, court tout au long de la controverse évoquée ici. 


\section{RÉFÉRENCES}

Abramson, A. 1987 The History of television, 1880 to 1941. Londres: Jefferson \& McFariand.

Beaune, C. 1985 Naissance de la nation France. Paris: Gallimard (Bibliothèque des Histoires).

Boltanski, L. et Thevenot L. 1987 Les Économies de la Grandeur. Paris: Cahiers du Centre d'études de l'emploi.

Bourdon, J. 1990 Histoire de la télévision sous de Gaulle. Paris: Anthropos et Institut national de l'audiovisuel.

Bourdon, J. \& M. Despratx 1988 Enjeux socio-professionnels de l'évolution des techniques de production à la télévision. Paris: Rapport au Ministère de la Recherche.

Chinaud, R. 1974 Rapport de la commission de contrôle de la gestion financière de l'O.R.T.F. Document parlementaire n¹072: Assemblée nationale séance, du 20 juin 1974.

Darre, Y. \& N. Heinich 1987 Cinéma et audiovisuel: professions et métiers. Paris: Service des études du ministère de la Culture.

Durieux, C. 1972 La Télécratie. Paris: Téma Éditions.

Goffman, E. 1975 Stigmates. Paris: Éditions de Minuit.

Latour, B. 1989 La Science en action. Paris: La Découverte.

Metz, C. 1971 Langage et Cinéma. Paris: Larousse.

— 1977 Le Signifiant imaginaire. Paris: UGE 10 : 18.

Morin, E. 1956 Le Cinéma ou l'homme imaginaire. Paris: Éditions de Minuit.

Perriault, J., 1978 Mémoires de l’ombre et du son, une archéologie de l'audiovisuel. Paris: Flammarion.

Thomas, I. \& Méadel, C. 1992 De la télévision à haute définition. Rapport non publié, Paris: Association Descartes et Centre de Sociologie de l'Innovation, École des Mines.

Thonon, M., C. Bec, M. Benard \& al. (1990) Les Réalisateurs, Rapport non publié. Paris: CNC et St Denis : Université Paris 8. 


\section{RÉSUMÉ}

Film ou vidéo, réflexion sur la pérennité d'une controverse technique. De nos jours encore, l'opposition entre cinéma et télévision est l'une des lignes de fracture autour desquelles se construit le débat culturel. Dans cet article, l'auteur montre que, loin d'être l'apanage des journalistes et du grand public, cette question a organisé dès l'origine les débats internes au milieu de la télévision sous la forme d'une opposition entre différentes techniques de production basées sur des supports distincts, le film et la vidéo: cette controverse a suscité l'émergence de nouveaux dispositifs techniques qui tentent, en vain, de dégager un compromis entre les parties en présence et a été finalement réifiée sous la forme d'associations durables entre des genres télévisuels et des modes de production.

\section{ABSTRACT}

Film or video, reflexions on the lonevity of a technological controversy. In present times the opposition between television and cinema is still a fracture line about which is centered a cultural debate. In this article the author shows that, far from being a prerogative of journalists and the public, this question has structured from the beginning discussions within television circles in the shape of an opposition between different production techniques based on different material bases, film and video : this argument has been at the origin of new technical systems which have tried in vain to reach a compromise reconciling the two opposing parties and has finally been objectified in the form of lasting associations between television types and productions means.

\section{MOTS CLÉS}

Film, vidéo, télévision, techniques de production, métiers de la télévision, réalisateurs, ingénieurs.

\section{KEYWORDS}

Film, video, television, production techonlogy, television professions, directors, engineers. 